\title{
Adult onset Still's disease presenting as fever of unknown origin: a case report with review of literature
}

\author{
Harpreet Singh, Deepak Jain, Saroj Dhankhar, Rekha Mathur \\ Department of Medicine and Division of Rheumatology, Pt. B.D. Sharma University of Health Sciences, Rohtak, India
}

\begin{abstract}
Adult onset Still's disease (AOSD) is a systemic inflammatory disease with exact etiology and pathogenesis yet to be discovered. AOSD, being an important cause of fever of unknown origin, is diagnosed after ruling out infections, malignancy and other rheumatologic diseases. It may present with fever without typical rash although typical triad is of fever, joint pain and rash. A 35-year-old previously healthy man was referred to our hospital with 6 months of fever, joint pain and weight loss. Examination and investigations revealed anemia, leukocytosis (predominant neutrophilia), lymphadenopathy, hepatomegaly, arthritis and evidence of interstitial lung disease with raised serum ferritin levels. Hematological disorders, infections and other rheumatologic diseases were excluded. The diagnosis of adult onset Still's disease can be very difficult as there are no specific tests and diagnosis is based on symptom complex. AOSD presenting as fever of unknown origin could be a challenge for the physician to diagnose and manage timely.
\end{abstract}

\section{Introduction}

Adult onset Still's disease (AOSD) is a systemic inflammatory disorder with reported incidence of $0.14-0.40$ per $100,000 .{ }^{1}$ It was initially described in 1897 by George F. Still. The disease has a bimodal age distribution with first peak between 15-25 years of age and second between 36-46 years of age. ${ }^{2}$ The exact etiological factors are still unknown, genetic and infectious etiology has been suggested in view of prodromal symptoms and role of non-specific cytokines like interleukin (IL)-5, IL-6, IL-18 and tumor necrosis factor-alpha (TNF- $\alpha$ ). ${ }^{3}$ Quotidian or double quotidian

Correspondence: Deepak Jain, Department of Medicine, Pt. B.D. Sharma University of Health Sciences, Rohtak-124001 (Haryana), India.

Tel.: +91.9416147887. E-mail: jaindeepakdr@gmail.com

Key words: Adult onset still's disease; fever of unknown origin; arthritis; leukocytosis.

Conflict of interest: the authors declare no conflict of interest.

Received for publication: 5 December 2016.

Revision received: 27 March 2017.

Accepted for publication: 12 April 2017.

This work is licensed under a Creative Commons Attribution NonCommercial 4.0 License (CC BY-NC 4.0).

CCopyright H. Singh et al., 2017

Licensee PAGEPress, Italy

Italian Journal of Medicine 2017; 11:388-392

doi:10.4081/itjm.2017.824 fever, arthralgia or arthritis, evanescent skin rash, sore throat, weight loss, lymphadenopathy and hepatosplenomegaly are the clinical manifestations of Still's disease. It is considered as a diagnosis of exclusion and only after excluding infections, malignancies and connective tissue diseases, diagnosis of AOSD is made based on the Yamaguchi or Fautrel criteria. ${ }^{4}$ Here, we report a case of AOSD, who presented as a case of fever of unknown origin (FUO).

Patient consent form was procured prior to the case report study.

\section{Case Report}

A 35-year-old, previously healthy male, was admitted with a six-month history of high grade fever, weight loss, myalgia, fatigue and generalized arthralgia involving shoulders, elbows, wrists, proximal interphalangeal joints, knees and ankles. He had morning stiffness and limitation of movement in involved joints due to pain. He had visited multiple hospitals before coming here, where he had already been treated with doxycycline, amoxicillin+clavulanic acid and injectable piperacillin-tazobactam but patient did not show any improvement in fever and joint pain.

On clinical examination, patient was conscious and well oriented. His blood pressure was 120/74 $\mathrm{mmHg}$, pulse rate: $110 / \mathrm{min}$ and body temperature: $102^{\circ} \mathrm{F}$. Patient was anemic but did not have any icterus, cyanosis or pedal edema. Painless, soft, mobile and non-matted lymph nodes of size $1 \mathrm{~cm}$ were palpable in posterior cervical region. Liver was palpable $1 \mathrm{~cm}$ below right costal margin in mid-clavicular 
line. Spleen was not palpable. On respiratory examination, bilateral infrascapular late-inspiratory crepitations were audible. Cardiovascular and neurological examinations were essentially normal. Both knee and wrist joints were swollen and tender upon palpation.

Complete hemogram revealed persistent leukocytosis with neutrophilia and normocytic normochromic anemia with hemoglobin $(\mathrm{Hb}): 8.8 \mathrm{~g} / \mathrm{dL}$, total leucocytes count: $16,000 / \mathrm{cu} \mathrm{mm}$ with $90 \%$ neutrophils. Liver function tests, renal function tests, serum electrolytes, thyroid function tests, lipid profile were within normal range. Patient had hypoalbuminemia and increased globulin levels. Urine and stool complete examination was normal. Urine culture and repeated blood cultures taken during fever spike were sterile. Various other biochemical parameters included: erythrocyte sedimentation rate (ESR) was $120 \mathrm{~mm} / \mathrm{1}^{\mathrm{st}} \mathrm{h}, \mathrm{C}$-reactive protein (CRP) positive, Mantoux test non-reactive, viral markers $\mathrm{HIV} / \mathrm{HbsAg}$ /anti-hepatitis $\mathrm{C}$ virus antibodies were non-reactive. Immunoglobulin (Ig) M for Leptospira, scrub typhus, Widal test, malaria antigen card test and IgM and IgG for Brucella were negative. All biochemical parameters for connective tissue diseases including rheumatoid factor (RF), anti-cyclic citrullinated peptide antibody, anti-nuclear antibody (ANA) by indirect fluorescence assay, cytoplasmic and perinuclear antineutrophil cytoplasmic antibodies were negative. Serum iron was $25.2 \mu \mathrm{g} / \mathrm{dL}$, total iron binding capacity: 156 $\mu \mathrm{g} / \mathrm{dL}, \%$ transferrin saturation: $16.15 \%$ although serum ferritin was significantly raised to a value of 1207.8 $\mathrm{ng} / \mathrm{mL}$. Diffuse and homogeneous accumulation of ${ }^{18} \mathrm{~F}$-fluorodeoxyglucose was seen in the bone marrow, lymph nodes and spleen on positron emission tomography scan. Test for glycosylated ferritin was planned but could not be done because of financial constraints and unavailability of the test in our institution.

Electrocardiogram and chest X-ray revealed no abnormality. X-ray bilateral hands showed periarticular osteopenia (Figure 1). Ultrasonography of the abdomen showed hepatomegaly with liver span of 16 $\mathrm{cm}$. Echocardiogram showed no evidence of infective endocarditis but minimal pericardial effusion was seen. Contrast-enhanced computed tomography (CECT) of the chest revealed mild intra and inter lobular sub pleural septal thickening in bilateral basal segments suggestive of early interstitial lung disease and mild pericardial effusion (Figure 2). CECT abdomen showed hepatomegaly $(17.5 \mathrm{~cm})$ and splenomegaly. Magnetic resonance imaging lumbosacral spine with whole spine screening showed no evidence of Pott's spine. Spirometry showed evidence of restrictive lung disease [forced vital capacity (FVC) $=47 \%$ and forced expiratory volume in the first second (FEV1) $=46 \%$ of predicted; FEV1/FVC=101\%).

Bone marrow examination was done which revealed nonspecific myeloid reaction with increased iron stores. Fine needle aspiration cytology cervical lymph nodes showed reactive lymphadenitis without any evidence of granuloma (Figure 3 ).

As per examination and tests, he was diagnosed as AOSD as per Yamaguchi criteria after ruling out all infections, neoplastic and other possible non-infectious inflammatory conditions. Non-steroidal anti-inflammatory drugs (NSAIDs) and prednisone were started. Patient improved dramatically and his fever as well as joint pain subsided. Patient was discharged in stable condition and is on regular follow-up.

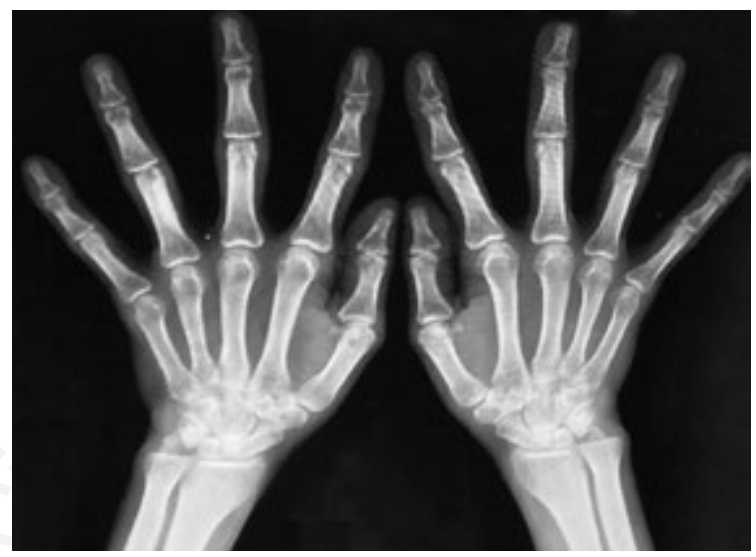

Figure 1. X-ray bilateral hands showing periarticular osteopenia.

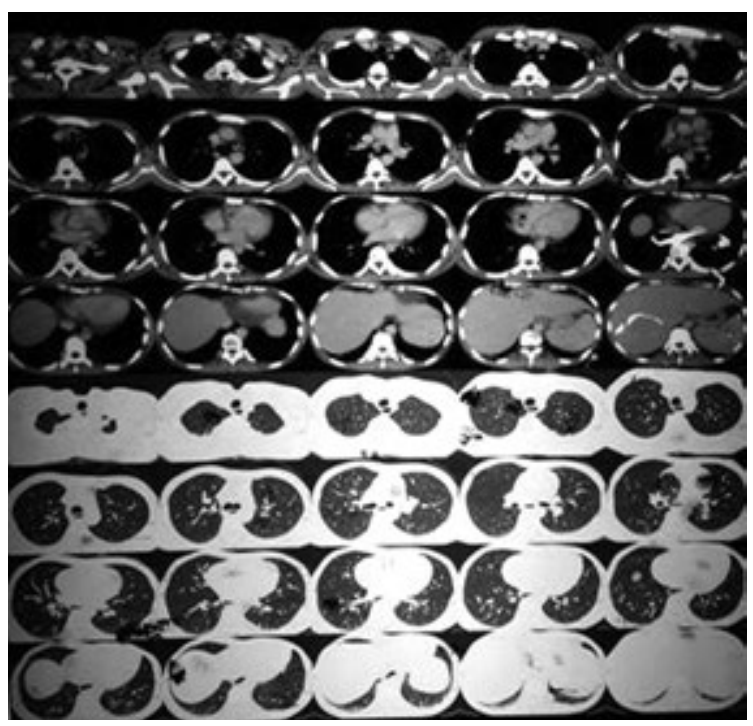

Figure 2. Contrast-enhanced computed tomography of the chest revealing mild intra- and inter-lobular subpleural septal thickening in bilateral basal segments suggestive of early interstitial lung disease and mild pericardial effusion. 


\section{Discussion}

Infections constitute most common etiology of FUO $(43 \%) .23 \%$ cases of FUO are caused by non-infectious inflammatory diseases among which AOSD accounts for $6 \%$ of cases with fever of unknown origin. $16 \%$ of cases with FUO are due to neoplasms, $4 \%$ of miscellaneous causes and the other $13 \%$ remains undiagnosed even after extensive investigations. ${ }^{5}$ AOSD is a disease of exclusion and a systemic auto inflammatory disease with infections and genetic factors as possible etiology. It is characterized by spiking fever, evanescent rash, articular manifestations and multiorgan involvement. ${ }^{6}$ AOSD has no pathognomonic features so diagnosed as per Yamaguchi's criteria which require the presence of at least five features with at least two of these being major criteria after ruling out infectious, malignant and other rheumatic diseases ${ }^{7}$ (Table 1). Other criteria like Fautrel's criteria are also proposed and are frequently used but none of them is fully validated. ${ }^{8}$ Our patient had high grade fever, arthralgia and neutrophilic leukocytosis from major criteria and hepatomegaly, lymphadenopa-

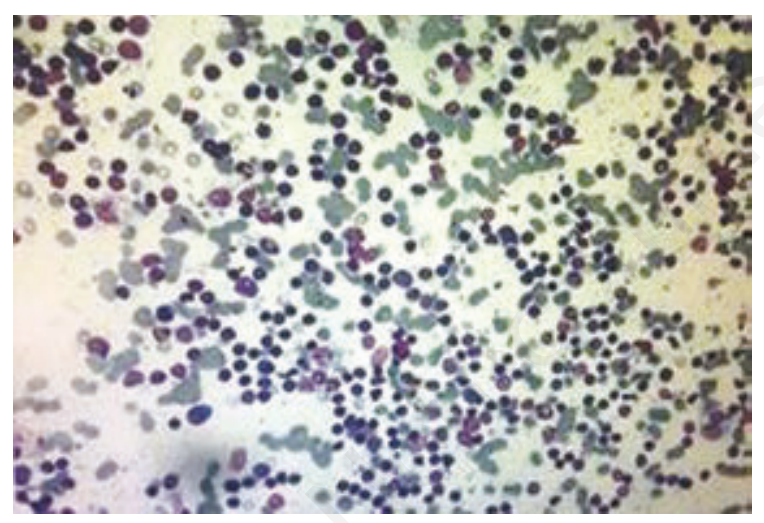

Figure 3. Fine needle aspiration cytology cervical lymph nodes showing reactive lymphadenitis without any evidence of granuloma. thy and RF and ANA negativity from minor criteria with no evidence of any infection, malignancy or other rheumatic disease even after detailed workup.

Fever is noted in more than $95 \%$ of cases and it is usually higher than $39^{\circ} \mathrm{C}$, one or more spikes daily or it may be continuous. ${ }^{6}$ Around $90 \%$ of patients have also sore throat due to non-exudative pharyngitis. Most patients also complain of significant weight loss. Characteristic Still's rash is evanescent and non-itchy which usually appears or enhances during fever spikes. Koebner's phenomenon and dermatographism are usually evident. ${ }^{9}$ Arthritis, commonly polyarthritis occurs in about $95 \%$ of cases, joint symptoms increase during fever spike. Most common joints involved are knees, followed by wrists, ankles, elbows, metacarpophalangeal, metatarsophalangeal, distal interphalangeal joints, hip, neck and temporomandibular joint and interphalangeal joints of the feet. ${ }^{10}$ Radiographs of affected joints in early disease show juxta-articular osteopenia and soft tissue swelling. Later in established arthritis, erosions and joint space narrowing may be detected. Selective non-erosive narrowing of carpometacarpal and intercarpal joint spaces leading to intercarpal ankylosis is more specific for AOSD. ${ }^{11}$

The disease may have monocyclic (25-30\%), polycyclic (25-30\%) or chronic course (30-50\%). In monocyclic course, disease remits within a year and never appears again. Those with polycyclic course have intermittent disease and intensity tends to be less severe and of shorter duration with subsequent episodes. Chronic disease is most disabling and leads to chronic disabling arthritis. The presence of polyarthritis earlier in disease course, involvement of hip or shoulder joints, prior episode in childhood and requirement of more than 2 years of treatment with steroids are poor prognostic factors. On the other hand, the absence of Still's rash predicts a better outcome. ${ }^{10}$

Lymphadenopathy, splenomegaly and hepatomegaly are detected in $63 \%, 52 \%$ and $42 \%$ of patients respectively. ${ }^{6,10}$ Lymphadenopathy and organomegaly may be observed as a result of reticuloendothelial involvement. Cervical lymph nodes are most commonly involved. About a third of patients

Table 1. Yamaguchi criteria for adult onset Still's disease. Presence of five features with at least two being major criteria are required for diagnosis.

\begin{tabular}{ll}
\hline Major criteria & Minor criteria \\
\hline Fever of at least $39^{\circ} \mathrm{C}$ for $\geq 1$ week & Sore throat \\
\hline Arthralgia $\geq 2$ weeks & Lymphadenopathy \\
\hline Evanescent rash during fever spike & Hepatomegaly or splenomegaly \\
\hline Leukocytosis $(>10,000)$ with neutrophilia $(>80 \%)$ & Negative for ANA and rheumatoid factor \\
\hline
\end{tabular}

ANA, anti-nuclear antibody; LFT, liver function tests. 
have pleural effusion and $27 \%$ of patients have pneumonitis affecting the upper or lower lobes. Rarely, patients develop acute respiratory distress syndrome and interstitial lung disease. Our patient also showed evidence of interstitial lung disease, which has been found as an association in around 5-6\% cases with Still's disease. Pericarditis and mild pericardial effusion are seen in about one third of patients, and very rarely they may lead to cardiac tamponade. Nearly $75 \%$ of patients have elevated liver enzymes which might be a result of drug toxicity or mostly primary. Leukocytosis is the result of a striking neutrophilia that is secondary to bone marrow granulocyte hyperplasia and seen in $92 \%$ of cases. Raised ESR is found in $99 \%$, hypoalbuminemia in $81 \%$, anemia of chronic disease in $65 \%$ and thrombocytosis in $62 \%$ of cases. ${ }^{10}$

Serum ferritin and glycosylated ferritin levels are considered to be specific diagnostic criteria for AOSD. S ferritn level $\geq 5$ upper limit of the normal has $80 \%$ sensitive and $46 \%$ specific for diagnosis. ${ }^{12,13}$ Levels of serum ferritin correlate with disease activity and are used for monitoring response. Glycosylation is a process that protects serum ferritin from proteolytic enzymes. Healthy subjects have $50-80 \%$ of serum ferritin glycosylated, whereas this fraction falls to $20-50 \%$ in patients with inflammatory diseases due to saturation of glycosylation mechanisms and by lower clearance of non-glycosylated proteins by histiocyte-macrophage system. ${ }^{14}$ So the percentage of glycosylated serum ferritin remains low throughout the course, both during active disease or remission and considered more specific for diagnosis of AOSD. ${ }^{8}$

Because of their similar clinical presentations and temporal relationships, Infections and malignancies have been suspected as etiologies of AOSD. However, a causal relationship has never been clearly established. Various infectious agents like viruses and bacteria may trigger AOSD. ${ }^{15}$ Some studies have looked at distinct HLA alleles in their association with AOSD, however no consistent results have been obtained from association studies between AOSD and HLA loci. Abnormalities in cytokines profile (IL-1, IL-6, IL-18, TNF- $\alpha$, macrophage colony stimulating factor, interferon gamma) may play a pathophysiological role in AOSD. ${ }^{16}$ Mainly IL-18 plays a crucial role in activating macrophages favoring Th- 1 type cytokine production. IL-18 and serum ferritin levels correlate with disease activity in AOSD. ${ }^{15,17}$ Rheumatoid factor and ANA are found negative in $93 \%$ and $92 \%$ of cases respectively.

Despite increasing progress in unraveling the pathogenesis of the disease, evidence-based data are still lacking and, consequently, the disease course is unpredictable at onset and the therapeutic approach is still largely anecdotal higher systemic score and/or the presence of AOSD-related complications at the time of diagnosis are significantly associated with a poor, unfavorable outcome. ${ }^{12}$ Treatment of AOSD centers around the use of NSAIDs, steroids and antirheumatic agents to control fever, arthritis and systemic disease. NSAID monotherapy controls the disease in only $7-15 \%$ of patients; others require steroids and show response in $76-95 \%$ of cases. ${ }^{18}$ Myocarditis, pericardial tamponade, disseminated intravascular coagulation and increasing liver enzymes on treatment with NSAIDs are absolute indications for steroids. With improvement in disease and inflammatory parameters dose of steroids needs to be tapered to minimum. Chronic polyarthritis which is not controlled with low dose steroids requires the use of antirheumatic drugs like methotrexate, hydroxychloroquine, cyclosporine A, gold, penicillamine, azathioprine and cyclophosphamide. Chimeric antiTNF monoclonal antibody infliximab used in nonresponsive patients at weeks $0,2,6$ and every 8 weeks shows marked improvement in symptoms and parameters like ESR, CRP, serum ferritin. ${ }^{19}$ Serious side effects including infusion reactions, cutaneous eruptions, recurrent bronchitis, pneumonitis, heart failure, blurry vision and fulminant hepatitis in patient with concomitant hepatitis B may be observed. Intravenous immunoglobulin use led to treatment of flares as well as remission in NSAID refractory patients. In an era of biologic medicines, promising targets like IL-1, IL-6, IL-17 have emerged as an essential aspect of the future prospects in the treatment of AOSD. ${ }^{15}$ IL-1 antagonist anakinra has been tried in refractory AOSD or those requiring high dose maintenance steroids and found to be beneficial. Antihuman IL-6 monoclonal antibody has shown marked improvement in arthralgia, CRP and fever in patients who are refractory to methotrexate, cyclosporin A and prednisolone..$^{20}$

\section{Conclusions}

The fever of unknown origin is often frustrating both to physician and patient as extensive invasive and noninvasive investigations may be required and sometimes even fail to reach a diagnosis. Hence for an effective and sensible approach it is very important that clinician considers noninfectious disease like Still's disease in differential diagnosis of FUO. Although Still's disease may require standard diagnostic criteria for diagnosis, it needs extensive investigations to exclude other possible diagnosis. High index of suspicion supported by relevant investigations is much needed for diagnosis of Still's disease so that early initiation of targeted therapy can be done to prevent poor outcome. 


\section{References}

1. Mahroum N, Mahagna H, Amital H. Diagnosis and classification of adult Still's disease. J Autoimmun 2014;4849:34-7.

2. Gerfaud-Valentin M, Jamilloux Y, Iwaz J, Seve P. Adult onset Still's disease. Autoimmun Rev 2014;13:708-2

3. Efthimiou P, Paik PK, Bielory L. Diagnosis and management of adult onset Still's disease. Ann Rheum Dis 2006;65:564-72.

4. Maset M, Costa EG, Carniello GS, et al. Fever and erythema: exclude all and then... think of Still's disease! Ital J Med 2016;10:245-49.

5. Manders CM, Simon A, Rovers CB. Fever of unknown origin. Clin Med 2015;15:280-4.

6. Iliou C, Papagoras C, Tsifetaki N, et al. Adult onset Still's disease: clinical, serological and therapeutic considerations. Clin Exp Rheumatol 2013;31:47-52.

7. Yamaguchi M, Ohta A, Tsunematsu T, et al. Preliminary criteria for classification of adult Still's disease. J Rheumatol 1992;19:424-30.

8. Fautrel B, Zing E, Golmard JL, et al. Proposal for a new set of classification criteria for adult onset still's disease. Medicine 2002;8:194-200.

9. Cozzi A, Papagrigoraki A, Biasi D, et al. Cutaneous manifestations of adult onset Still's disease: a case report and review of the literature. Clin Rheumatol 2016;35: 1377-82.

10. Pouchot J, Sampalis JS, Beardet F, et al. Adult Still's disease: manifestations, disease course and outcome in 62 patients. Medicine (Baltimore) 1991;70:118-36.

11. Bjorkengren AG, Pathria MN, Sartoris DJ, et al. Carpal alterations in adult onset Still's disease, juvenile chronic arthritis and adult onset rheumatoid arthritis: a comparative study. Radiology 1987;165:545-8.

12. Ruscitti P, Cipriani P, Masedu F, et al. Adult-onset Still's disease: evaluation of prognostic tools and validation of the systemic score by analysis of 100 cases from three centers. BMC Med 2016;14:194.

13. VanReeth C, Lemoel G, LasneYetal. Serum ferritin and isoferritins are tools for diagnosis of active adult still's disease. J Rheumatol 1994;21:890-5.

14. Wouters JM, Vander Veen J, VandePutte LB, De Rooji DJ. Adult onset Still's disease and viral infections. Ann Rheum Dis 1988;47:764-7.

15. Siddiqui M, Putman M, Dua AB. Adult-onset Still's disease: current challenges and future prospects. Open Access Rheumatol Res Rev 2016;8:17-22.

16. Qinjin H, Zhencheng Y, Jian Z. Adult onset Still's disease:how to make adiagnosis in an atypical case. Rheumatol Int 2012;32:3299-302.

17. Ichida H, Kawaguchi Y, Sugiura T, et al. Clinical manifestations of adult onset Still's disease presenting with erosive arthritis: association with low levels of ferritin and IL-18. Arthritis Care Res 2014;66:642-6.

18. Masson C, Le Loet X, Liote F, et al. Adult Still's disease. Part II. Management, outcome and prognostic factors. Rev Rhum Engl Ed 1995;62:758-65.

19. Efthimiou P, Markenson JA. Role of biological agents in immune mediated inflammatory diseases. South Med J 2005;98:192-204.

20. Iwamoto M, Nara H, Hirata D, et al. Humanised monoclonal anti interleukin- 6 receptor antibody for treatment of intractable adult onset Still's disease. Arthritis Rheum 2002;46:3388-9. 